\title{
Stage IV Oropharyngeal Carcinoma AJCC v6
}

National Cancer Institute

\section{Source}

National Cancer Institute. Stage IV Oropharyngeal Carcinoma A/CC v6. NCI Thesaurus.

Code $C 9218$.

Stage IV includes: IVA (T4a, N0, M0); (T4a, N1, M0); (T1, N2, M0); (T2, N2, M0); (T3, N2, M0); (T4a, N2, M0); IVB (T4b, Any N, M0); IVC (Any T, Any N, M1). T4a: T umor invades the larynx, deep/extrinsic muscle of tongue, medial pterygoid, hard palate, or mandible. T4b: Tumor invades lateral pterygoid muscle, pterygoid plates lateral nasopharynx, or skull base or encases carotid artery. M1: Distant Metastasis. (AJCC 6th ed.) 